\title{
microRNA-20a enhances the epithelial-to-mesenchymal transition of colorectal cancer cells by modulating matrix metalloproteinases
}

\author{
TAO XU, CHANGQING JING, YULONG SHI, RUIZHENG MIAO, \\ LIPAN PENG, SHUAI KONG, YAN MA and LEPING LI
}

\begin{abstract}
Department of Gastrointestinal Surgery, Provincial Hospital Affiliated to Shandong University, Jinan, Shandong 250021, P.R. China
\end{abstract}

Received August 2, 2014; Accepted April 13, 2015

DOI: $10.3892 /$ etm.2015.2538

\begin{abstract}
The mortality rates associated with colorectal cancer (CRC) are high due to metastasis. Epithelial-to-mesenchymal transition (EMT) is a key step in tumor metastasis. The aim of the present study was to investigate the function of microRNA-20a (miR-20a) in EMT. The expression of miR-20a was analyzed in CRC tissues and cell lines using the reverse transcription-quantitative polymerase chain reaction. Plasmids containing miR-20a short hairpin RNA and miR-20a mimics were transfected into SW620 and LS174T cell lines, respectively. Cell counting kit-8, Transwell ${ }^{\circledR}$ and wound healing assays were performed to assess the effects of miR-20a on cell proliferation, invasion and migration. EMT markers and matrix metalloproteinases (MMPs) were identified using western blotting. The results showed that increased expression of miR-20a in CRC tissues was associated with tumor invasion and lymph node metastasis $(\mathrm{P}<0.05)$. Further experiments indicated that miR-20a-knockdown inhibited the proliferation, invasion and migration of CRC cells, upregulated the expression of vimentin and tissue inhibitor of metalloproteinases- 2 (TIMP-2) and downregulated the expression of E-cadherin, MMP-2 and MMP-9. The opposite effects were observed in CRC cell lines overexpressing miR-20a. In conclusion, these results have shown that the upregulation of miR-20a suppresses TIMP-2 expression, which subsequently increases the expression of MMP-2 and MMP-9, thereby promoting the EMT of CRC cells. These findings suggest that miR-20a represents a potential therapeutic target for patients with CRC.
\end{abstract}

Correspondence to: Professor Leping Li, Department of Gastrointestinal Surgery, Provincial Hospital Affiliated to Shandong University, 324 Jingwu Road, Jinan, Shandong 250021, P.R. China E-mail: lep_li@163.com

Key words: microRNA-20a, epithelial-to-mesenchymal transition, colorectal cancer

\section{Introduction}

According to the GLOBOCAN 2012 statistics, colorectal cancer (CRC) is the third most common cancer in the world. Every year there are $\sim 0.7$ million CRC-related mortalities worldwide (1). The usual treatments for CRC are surgery, radiotherapy, chemotherapy and drugs; however, the therapeutic effects of these strategies are insufficient due to the occurrence of invasion and metastasis (2). Tumor metastasis is a complicated process involving multiple steps, one of which is known as the epithelial-to-mesenchymal transition (EMT). As a result of the EMT, epithelial cells gain a mesenchymal phenotype, enabling them to migrate to and invade a distant organ (3). An enhanced understanding of the molecular mechanisms regulating the EMT in CRC is therefore critical for more effective treatments.

microRNAs (miRNAs) are small, single-stranded RNA molecules of 20-24 bases in length that belong to the non-coding RNA species. miRNAs regulate gene expression by binding to the 3'-untranslated region (UTR) of their target mRNA, inhibiting translation or degrading the mRNA. To date, $>1,400$ miRNAs have been identified, and >5,300 human genes have been predicted to be targets of miRNAs (4). miRNAs are involved in numerous biological processes, including embryonic development, cell differentiation and metabolism (5-7). Aberrant miRNA expression in cancer has been found to participate in several pathological processes, such as EMT and other events contributing to tumorigenesis $(8,9)$.

miR-20a is a member of the miR-17-92 cluster, which includes miR-17-5p, miR-17-3p, miR-18a, miR-19a, miR-20a, miR-19b-1 and miR-92-1, and is located on chromosome $13 q 31$ (10). miR-20a has been reported to be associated with EMT in various types of cancers. In hepatocellular carcinoma (HCC), miR-20a restoration has been shown to inhibit cell proliferation and induce apoptosis by targeting the 3'-UTR of Mcl-1, while decreased expression of miR-20a has been reported to correlate significantly with the aggressive characteristics of HCC cells (11). In addition, the upregulated expression of miR-20a has been found to decrease the migratory ability of oral squamous cell carcinoma (OSCC) cell lines (12), and the ectopic expression of miR-20a has been demonstrated to induce the EMT and enhance the metastasis of gallbladder carcinoma (GBC) cells in vitro and in vivo (13). 
In CRC, however, the role of miR-20a in EMT remains largely unknown. The aim of the present study, therefore, was to characterize the expression profile of miR-20a in CRC tissues and analyze its correlation with the clinicopathological characteristics of the condition, and then to clarify the role of miR-20a in EMT in CRC cells lines.

\section{Materials and methods}

Tissue samples and cell culture. The present study was approved by the Committee for the Ethical Review of Research at the Provincial Hospital affiliated to Shandong University (Jinan, China). A total of 30 samples of tumor and adjacent non-tumor tissue were obtained from the Provincial Hospital, and signed consent forms were obtained from all patients. The human SW620, SW480, LS174T and HCT116 CRC cell lines and the normal human colonic epithelial cell line FHC were purchased from the Cell Bank of Chinese Academy of Sciences (Shanghai, China). The cells were cultured in RPMI-1640 and Dulbecco's modified Eagle's medium (Gibco-BRL, Grand Island, NY, USA) supplemented with $10 \%$ fetal bovine serum (Gibco-BRL) at $37^{\circ} \mathrm{C}$ in a humidified atmosphere containing $5 \% \mathrm{CO}_{2}$.

Reverse transcription-quantitative polymerase chain reaction $(R T-q P C R)$. Total RNA was extracted using TRIzol ${ }^{\circledR}$ reagent (Invitrogen Life Technologies, Carlsbad, CA, USA) according to the manufacturer's instructions, and the miRNA was reverse-transcribed into miRNA using a One-Step PrimeScript ${ }^{\circledR}$ miRNA cDNA Synthesis kit (Takara Bio, Inc., Shiga, Japan). RT-qPCR was performed using a SYBR ${ }^{\circledR}$ Premix Ex Taq kit (Takara Bio, Inc.) on an ABI 7300 qPCR system (Applied Biosystems, Foster City, CA, USA). The primers for miR-20a and the reference gene U6 were purchased from RiboBio Co., Ltd. (Guangzhou, China) and were as follows: miR-20a, F 5'-TACGATAAAGTGCTTATAGTGCAGGTA G-3'; U6, F 5'-AAAGACCTGTACGCCAACAC-3'. The PCR cycle conditions consisted of an initial denaturation step at $95^{\circ} \mathrm{C}$ for $30 \mathrm{sec}$, followed by 40 cycles at $95^{\circ} \mathrm{C}$ for $30 \mathrm{sec}$ and $60^{\circ} \mathrm{C}$ for $1 \mathrm{~min}$, and a final extension step of $60^{\circ} \mathrm{C}$ for $1 \mathrm{~min}$. The relative expression ratio of miR-20a was quantified using the $2^{-\Delta \Delta C T}$ method.

Establishment of CRC cell lines with knocked down and overexpressed miR-20a. Plasmids containing miR-20a inhibitor, miR-20a mimics, inhibitor negative control and mimic negative control were purchased from GenePharma (Shanghai, China). The SW620 and LS174T cell lines were selected for knockdown and overexpression modifications, respectively. A total of $2 \times 10^{5}$ cells were seeded into a six-well plate. Twenty-four hours later, when the cells were $70-90 \%$ confluent, $2 \mu \mathrm{g}$ plasmids were transfected into the cells using Lipofectamine ${ }^{\circledR} 2000$ (Invitrogen Life Technologies). Stably transfected cell lines were selected using $400 \mu \mathrm{g} / \mu \mathrm{l} \mathrm{G} 418$ (Sigma, St. Louis, MO, USA) for 1 month.

Cell proliferation assay. Cell proliferation was measured using a cell counting kit-8 (CCK-8) assay (Dojindo Molecular Technologies, Kumamoto, Japan) according to the manufacturer's instructions.
Transwell $^{\circledR}$ assay. Cell invasion was measured using Transwell chambers (Corning, Inc., Corning, NY, USA). Cells were suspended in serum-free medium and seeded in the upper chamber; $500 \mu \mathrm{l}$ complete RPMI-1640 was added to the lower well. The cells were incubated for $24 \mathrm{~h}$ and the cells that invaded the lower surface were fixed with methanol for $10 \mathrm{~min}$ and stained with crystal violet (Beyotime Co., Shanghai, China) for $30 \mathrm{~min}$. The cells were counted under a microscope (Nikon, Tokyo, Japan) at a magnification of x400.

Wound healing assay. A total of $5 \times 10^{5}$ cells were seeded in a six-well plate and incubated overnight until the cells had grown to a confluent monolayer. The wound was then made by scratching with a 200- $\mu$ l Eppendorf tip, and the wounded monolayers were washed with phosphate-buffered saline twice to remove any non-adherent cells, prior to being incubated in serum-free medium. Images of the wound areas were captured under a microscope (Nikon).

Western blot analysis. Radioimmunoprecipitation assay lysis buffer and phenylmethylsulfonyl fluoride (Beyotime Co.) were added to the cells. After lysis for $30 \mathrm{~min}$, the cellular extracts were centrifuged at $13,000 \mathrm{x} \mathrm{g}$ for $10 \mathrm{~min}$ at $4^{\circ} \mathrm{C}$, and the supernatant was frozen at $-80^{\circ} \mathrm{C}$. The total extracted protein was quantified using a bicinchoninic acid protein assay kit (Beyotime Co.). The proteins were separated using $10 \%$ sodium dodecyl sulfate-polyacrylamide gel electrophoresis, and then transferred to polyvinylidene difluoride membranes and blocked in 5\% non-fat milk in Tris buffered saline/Tween 20 buffer. The membranes were incubated overnight at $4^{\circ} \mathrm{C}$ with primary antibodies against E-cadherin (\#14472, mouse monoclonal, 1:500), vimentin (\#3878, rabbit polyclonal, 1:500), matrix metalloproteinase-2 (MMP-2) (\#4022, rabbit polyclonal, 1:1,000) and MMP-9 (\#13667, rabbit monoclonal, 1:500), purchased from Cell Signaling Technology Inc. (Beverly, MA, USA), and mouse monoclonal primary antibodies against TIMP-2 (\#MAB3310, mouse monoclonal, 1:500, Chemicon International, Inc., Temecula, CA, USA) and $\beta$-actin (\#BM0627, 1:1,000, Wuhan Boster Biological Technology, Ltd., Wuhan, China). After the membranes were washed with TBST $6 \times 10 \mathrm{~min}$, the membranes were incubated with secondary antibodies (\#BA1054, goat anti-rabbit and \#BA1051, goat anti-mouse 1:2,000; Wuhan Boster Biological Technology, Ltd.). The protein bands were detected using an enhanced chemiluminescence detection system (Beyotime Co.). $\beta$-actin was used as a loading control.

Statistical analysis. Data are presented as the mean \pm standard deviation from at least three separate experiments. The correlations between miR-20a expression levels and pathological features were analyzed with the $\chi^{2}$ test. All statistical analyses were conducted using SPSS version 17.0 statistical software (SPSS, Inc., Chicago, IL, USA). $\mathrm{P}<0.05$ was considered to indicate a statistically significant difference.

\section{Results}

Increased miR-20a expression in CRC tissues and cell lines. RT-qPCR analysis was carried out to detect the level of miR-20a in 30 tumor and matched non-tumor tissues, four 

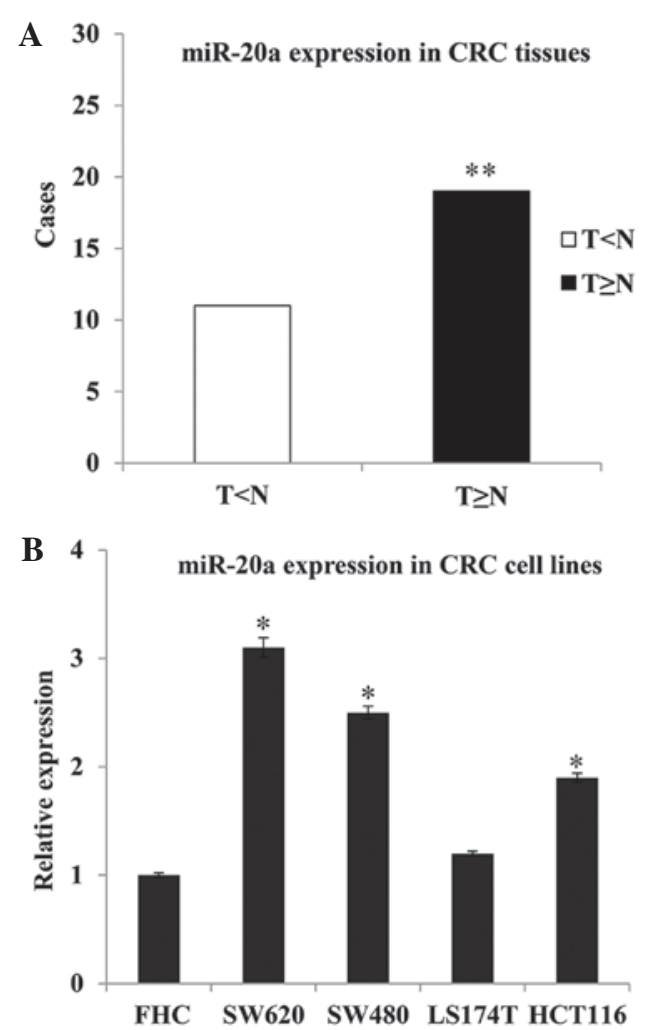

Figure 1. Relative expression level of miR-20a in CRC tissues and cell lines. The reverse transcription-quantitative polymerase chain reaction was used to detect the relative expression of miR-20a in CRC (A) tissues and (B) cell lines. U6 was used as an internal control. Error bars represent the mean \pm standard deviation from three independent experiments. ${ }^{*} \mathrm{P}<0.05$, ${ }^{* *} \mathrm{P}<0.01$. miR-20a, microRNA-20a; CRC, colorectal cancer; $\mathrm{T}$, tumor tissues; $\mathrm{N}$, adjacent non-tumor tissues.

CRC cell lines (SW620, SW480, LS174T and HCT116) and one normal colon epithelium cell line (FHC). The results showed an increased expression of miR-20a in the tumor tissues compared with the non-tumor tissues; the frequency of miR-20a upregulation was 63.33\% (19/30; $\mathrm{P}=0.008)$ (Fig. 1A). The four CRC cell lines also exhibited higher miR-20a expression levels than the normal colon epithelium cells (SW620 vs. FHC, $\mathrm{P}=0.016$; SW480 vs. FHC, $\mathrm{P}=0.021$; and HCT116 vs. FHC, $\mathrm{P}=0.024$ ) (Fig. 1B).

In order to explore the association between the expression pattern of miR-20a and the clinicopathological characteristics of the patients with CRC, 30 patients were categorized into low ( $<1.5$-fold) and high ( $\geq 1.5$-fold) miR-20a expression groups. The results are summarized in Table I. Statistical analyses revealed that the high expression of miR-20a was associated with tumor invasion $(\mathrm{P}=0.015)$ and lymph node metastasis $(\mathrm{P}=0.047)$, but not with age, gender, differentiation or distant metastasis. These data indicate that increased miR-20a expression plays an important role in CRC metastasis.

Levels of miR-20a in CRC cell lines with knocked down and overexpressed miR-20a. SW620 cells transfected with miR-20a inhibitor and inhibitor negative control plasmids were termed SW620-Si20a and SW620-NC cells, respectively. LS174T cells transfected with miR-20a mimic and mimic negative control plasmids were termed LS174T-OV20a and LS174T-NC cells, respectively. RT-qPCR was conducted
Table I. Association between miR-20a expression and the clinicopathological characteristics of patients with colorectal cancer.

\begin{tabular}{|c|c|c|c|}
\hline \multirow[b]{2}{*}{ Characteristic } & \multicolumn{2}{|c|}{$\begin{array}{l}\text { Relative miR-20a } \\
\text { expression }\end{array}$} & \multirow[b]{2}{*}{ P-value } \\
\hline & Low (n) & High (n) & \\
\hline \multicolumn{4}{|l|}{ Gender } \\
\hline Male & 8 & 11 & 0.466 \\
\hline Female & 3 & 8 & \\
\hline \multicolumn{4}{|l|}{ Age in years } \\
\hline$<60$ & 2 & 7 & 0.419 \\
\hline$\geq 60$ & 9 & 12 & \\
\hline \multicolumn{4}{|l|}{ Differentiation } \\
\hline Poorly & 3 & 7 & 0.702 \\
\hline Moderately/well & 8 & 12 & \\
\hline \multicolumn{4}{|l|}{ Local invasion } \\
\hline Yes & 7 & 3 & 0.015 \\
\hline No & 4 & 16 & \\
\hline \multicolumn{4}{|c|}{ Lymph node metastasis } \\
\hline Yes & 8 & 5 & 0.047 \\
\hline No & 3 & 14 & \\
\hline \multicolumn{4}{|l|}{ Distant metastasis } \\
\hline Yes & 6 & 4 & 0.108 \\
\hline No & 5 & 15 & \\
\hline
\end{tabular}

Differences between variables were assessed using the $\chi^{2}$ test. miR-20a, microRNA-20a.

to confirm the expression levels of miR-20a, and the results showed that, compared with the SW620-NC cells, the expression of miR-20a in the SW620-Si20a cells was decreased $(\mathrm{P}=0.031)$. By comparison, the LS174T-OV20a cells showed an increased level of miR-20a when compared with the LS174T-NC cells ( $\mathrm{P}=0.015)$ (Fig. 2).

miR-20a promotes the proliferative ability of colon cells. The CCK- 8 assay was employed to detect the proliferative ability of cells with knocked down or overexpressed miR-20a. Suppression of miR-20a significantly inhibited the growth of SW620 cells at the fourth day $(\mathrm{P}<0.05)$, while miR-20a overexpression enhanced LS174T cells proliferation at the same time-point $(\mathrm{P}<0.05)$ (Fig. $3 \mathrm{~A}$ and $\mathrm{B})$. These results indicate that miR-20a promotes the proliferative ability of CRC cell lines.

miR-20a enhances the invasive and migratory ability of $C R C$ cells. To evaluate the role of miR-20a in invasion and migration, Transwell and wound healing assays were performed using the stably transfected CRC cell lines. Compared with the control, SW620-Si20a cells exhibited a significantly inhibited invasive ability. By contrast, overexpression of miR-20a increased the invasion of LS174T-OV20a cells as compared with the LS174T-NC cells (Fig. 4A). Consistent 

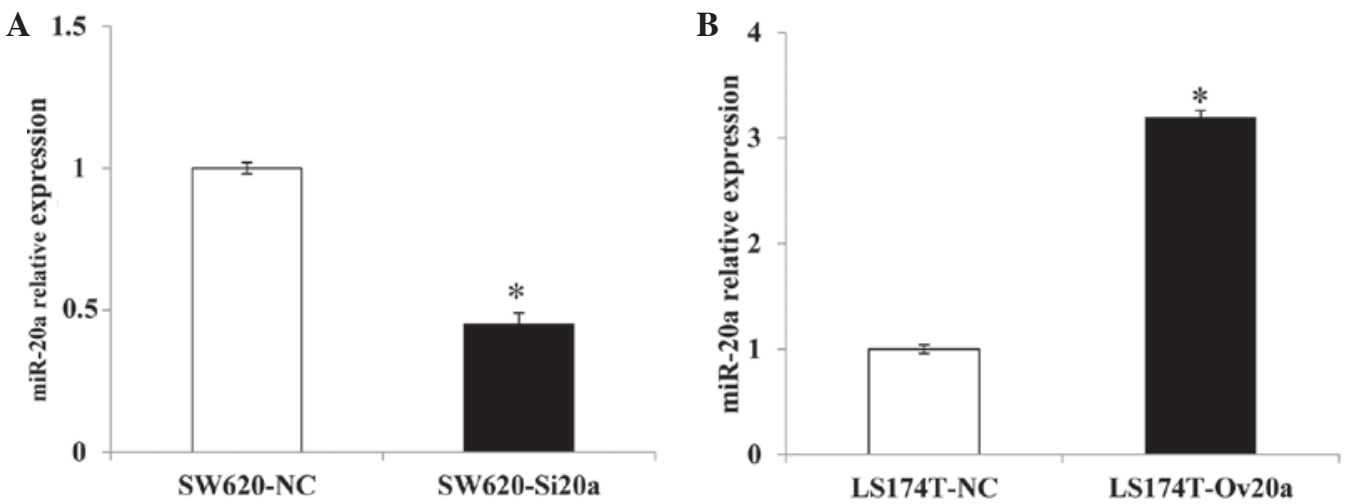

Figure 2. Expression level of miR-20a in knocked down and overexpressed colorectal cancer cell lines. The reverse transcription-quantitative polymerase chain reaction was used to detect the expression of miR-20a in (A) SW620-NC and SW620-Si20a and (B) LS174T-NC and LS174T-OV20a cell lines. U6 was used as an internal control. Error bars represent the mean \pm standard deviation from three independent experiments. "P $<0.05$. miR-20a, microRNA-20a; SW620-NC, SW620 cells transfected with miR-20a inhibitor negative control plasmid; SW620-Si20a, SW620 cells transfected with miR-20a inhibitor plasmid; LS174T-NC, LS174T cells transfected with miR-20a mimic negative control plasmid; LS174T-OV20a, LS174T cells transfected with miR-20a mimic plasmid.
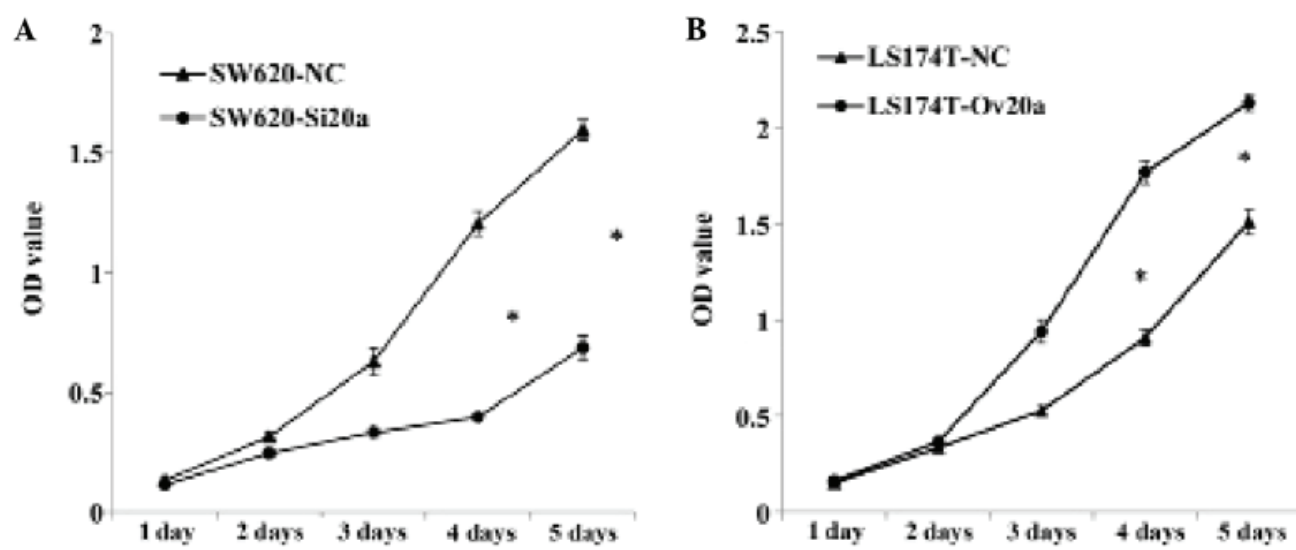

Figure 3. Effect of miR-20a on cell proliferation. The proliferative ability was detected by performing the cell counting kit- 8 assay using colorectal cancer cell lines with (A) knocked down and (B) overexpressed miR-20a. "P<0.05. miR-20a, microRNA-20a; OD, optical density; SW620-NC, SW620 cells transfected with miR-20a inhibitor negative control plasmid; SW620-Si20a, SW620 cells transfected with miR-20a inhibitor plasmid; LS174T-NC, LS174T cells transfected with miR-20a mimic negative control plasmid; LS174T-OV20a, LS174T cells transfected with miR-20a mimic plasmid.

with these results, the wound healing assay indicated that miR-20a-knockdown slowed the rate of wound closure, while miR-20a overexpression increased the migratory ability (Fig. 4B). Western blot analyses showed that the loss of miR-20a expression enhanced the level of vimentin and reduced the level of E-cadherin in SW620 cells; by contrast, ectopic expression of miR-20a in the LS174T cells led to an increased level of E-cadherin and a decreased level of vimentin (Fig. 4C and D). These findings demonstrate that miR-20a enhances the EMT of CRC cells.

miR-20a enhances EMT by modulating the expression of TIMP-2 and MMPs. The finding that miR-20a is involved in the promotion of cell invasion and migration suggested that miR-20a is additionally involved in the EMT process. To further explore the mechanism by which miR-20a modulated the EMT, western blot analysis was performed to examine the expression of EMT-related markers. In the SW620-Si20a cells, knockdown of miR-20a upregulated the TIMP-2 and downregulated the MMP-2 and MMP-9 protein levels (Fig. 5A). In the LS174T-OV20a cells, overexpression of miR-20a inhibited the expression of TIMP-2 and induced the expression of MMP-2 and MMP-9 (Fig. 5B). These data indicate that miR-20a enhances EMT by regulating the expression of TIMP-2, MMP-2 and MMP-9.

\section{Discussion}

In $\mathrm{CRC}$, the high mortality rates are primarily a result of tumor metastasis. At diagnosis, most patients are found to exhibit distant metastasis; even following surgery, $~ 50 \%$ of these patients are likely to undergo tumor relapse and succumb (14). The inhibition of tumor metastasis, therefore, is a promising therapeutic approach. Increasing numbers of miRNAs have been demonstrated to play significant roles in cell proliferation and the migration and invasion of various types of tumor, suggesting that these molecules represent a novel class of therapeutic target in cancer (8).

Studies have shown that miR-20a exhibits different expression profiles in different types of cancer (11-13,15-18). Upregulation of miR-20a has been found in GBC (13), colon adenocarcinoma (15) and gliomas (16), while miR-20a down- 
A

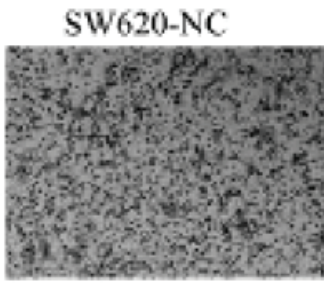

B
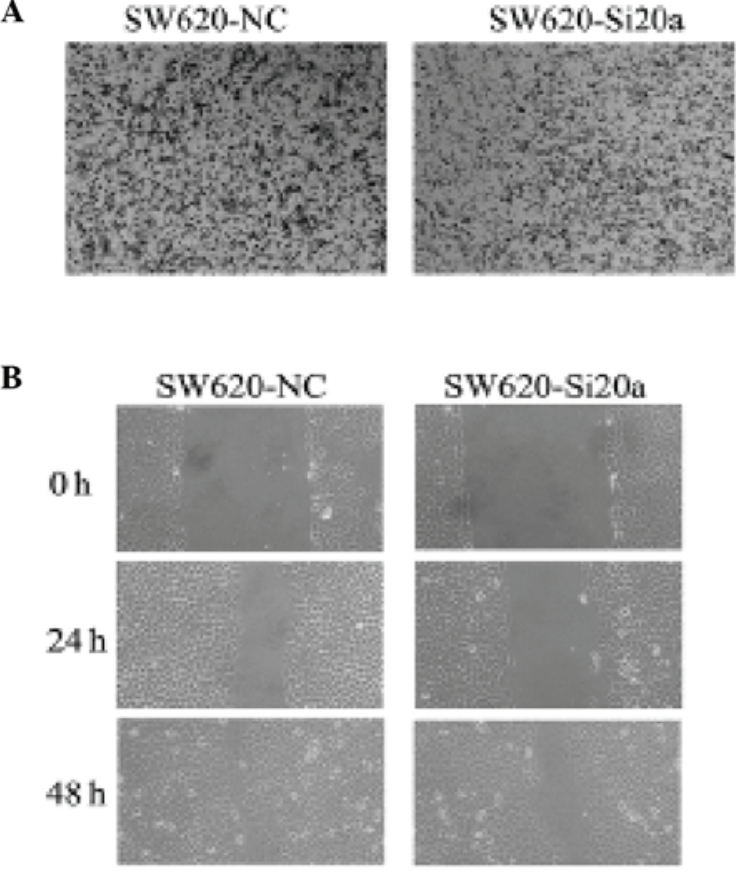

SW620-Si20a
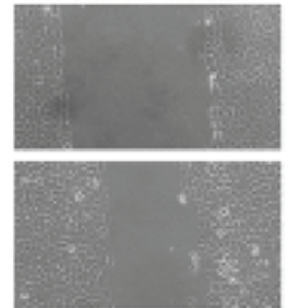

C
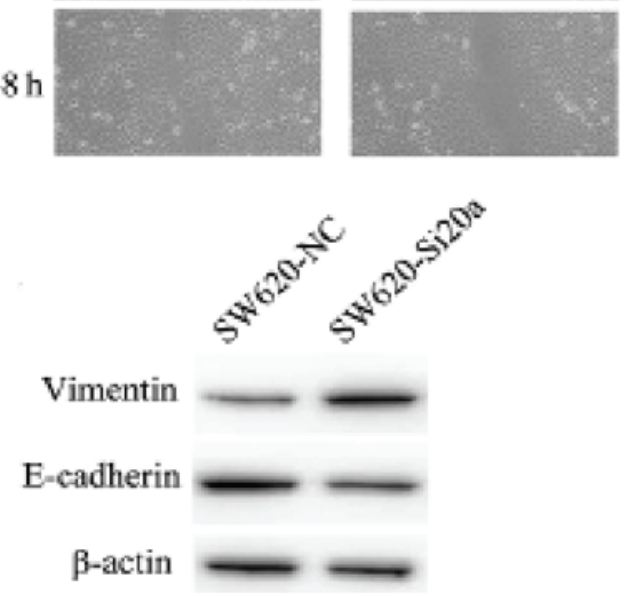

LS174T-NC

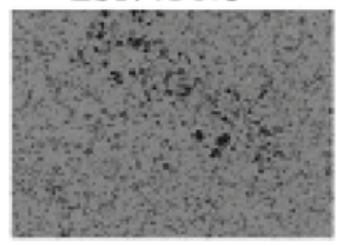

LS174T-NC

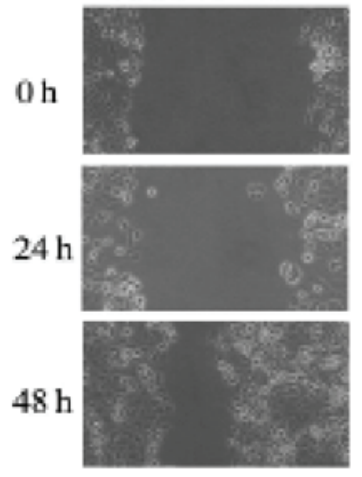

D

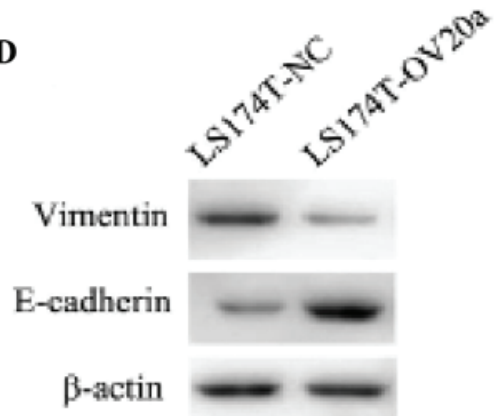

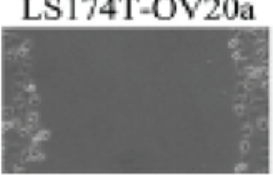

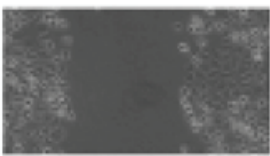

LS1 74T-OV20a
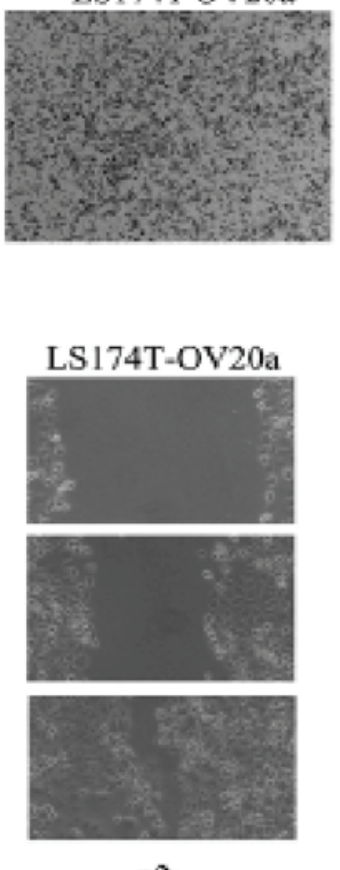

Figure 4. Effect of miR-20a on cell migration. (A) The Transwell ${ }^{\circledR}$ assay was used to assess the invasive ability of the SW620-NC, SW620-Si20a, LS174T-NC and LS174T-OV20a cell lines (x400). (B) A wound healing assay was used to assess the mobility of the SW620-NC, SW620-Si20a, LS174T-NC and LS174T-OV20a cell lines (x400). (C and D) The expression of the epithelial-to-mesenchymal transition markers E-cadherin and vimentin in the (C) SW620-NC and SW620-Si20a and (D) LS174T-NC and LS174T-OV20a cell lines was examined by western blot analysis. miR-20a, microRNA-20a; SW620-NC, SW620 cells transfected with miR-20a inhibitor negative control plasmid; SW620-Si20a, SW620 cells transfected with miR-20a inhibitor plasmid; LS174T-NC, LS174T cells transfected with miR-20a mimic negative control plasmid; LS174T-OV20a, LS174T cells transfected with miR-20a mimic plasmid.
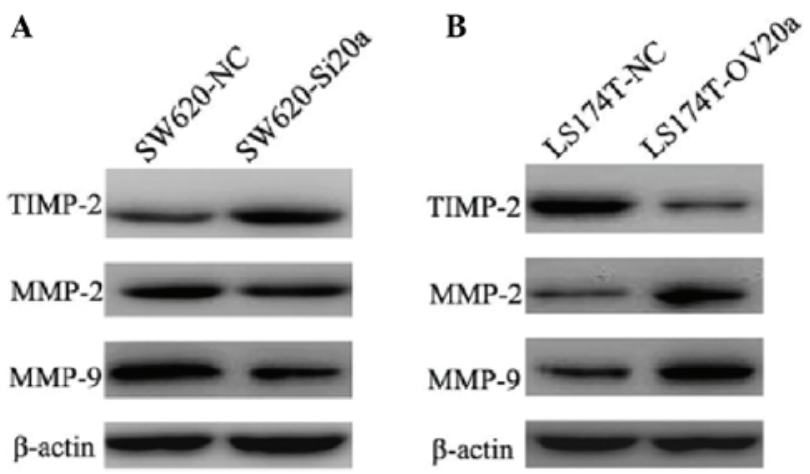

Figure 5. Expression of the epithelial-to-mesenchymal transition-related proteins. The effect of miR-20a on the protein expression of TIMP-2, MMP-2 and MMP-9 in colorectal cancer cell lines with (A) knocked down and (B) overexpressed miR-20a was analyzed using western blotting. miR-20a, microRNA-20a; TIMP2, tissue inhibitor of metalloproteinases-2; MMP, matrix metalloproteinase; SW620-NC, SW620 cells transfected with miR-20a inhibitor negative control plasmid; SW620-Si20a, SW620 cells transfected with miR-20a inhibitor plasmid; LS174T-NC, LS174T cells transfected with miR-20a mimic negative control plasmid; LS174T-OV20a, LS174T cells transfected with miR-20a mimic plasmid. regulation has been reported in primary HCC (11), OSCC (12), breast cancer (17) and pancreatic carcinoma (18). This suggests that the function of miR-20a may vary among different types of tumor. The present data showed an elevated expression profile of miR-20a in $30 \mathrm{CRC}$ cases, which is consistent with the data from a previous study (19). Further analysis revealed that the high expression of miR-20a in patients with CRC was associated with tumor invasion and lymph node metastasis. It should be noted, however, that the number of clinical samples in this study was relatively small; therefore, further research with larger samples is necessary to verify the findings. By investigating the biological function of miR-20a in CRC cells, the present study showed that miR-20a enhanced the proliferation, invasion and migration of the cells.

EMT is an biological process that can be classified into one of three subtypes: Embryogenesis and organ development, tissue regeneration and organ fibrosis or cancer progression and metastasis (20). During cancer progression, cancer cells detach from the basement membrane, migrate to and invade surrounding tissues and eventually colonize remote sites (21). 
A hallmark of cells that have undergone EMT is the loss of the epithelial cell marker E-cadherin and the gain of the mesenchymal cell markers N-cadherin and vimentin (22). Given that the present results revealed that miR-20a increased the EMT-related cell invasion and migration, the next step was to examine the changes in the markers of EMT. The epithelial marker E-cadherin was upregulated following transfection with the miR-20a mimics and decreased following transfection with the inhibitor of miR-20a. By comparison, the mesenchymal marker vimentin was downregulated following transfection with the miR-20a mimics and increased following transfection with the inhibitor of miR-20a. These results further confirmed that miR-20a was involved in modulating the EMT.

To further investigate the mechanism of miR-20a in enhancing EMT, the expression of TIMP-2, MMP-2 and MMP-9 was examined. MMP-2 and MMP-9 are members of the MMP family that degrade the basement membrane and extracellular matrix, facilitating the invasion and migration of cancer cells $(23,24)$. Aberrant expression of MMP-2 and MMP-9 has been confirmed to be associated with proliferation, invasion and EMT in a variety of types of cancer $(25,26)$. TIMP-2 is the key endogenous regulator of MMPs; by inhibiting the activity of MMPs, it thus inhibits tumor migration (27). The present data showed that, with the downregulation of miR-20a in SW620 cells, the expression of TIMP-2 was upregulated and that of MMP-2 and MMP-9 was downregulated. The opposite results were obtained in LS174T cells overexpressing miR-20a. Wang et al (28) found that TIMP-2 is the direct target of miR-20a; in the present study, therefore, miR-20a may have increased the expression of MMP-2 and MMP-9 by suppressing the expression of TIMP-2.

In conclusion, the results of the present study have demonstrated that miR-20a is upregulated in CRC tissues and that this upregulation is associated with tumor invasion and lymph node metastases $(\mathrm{P}<0.05)$. Furthermore, miR-20a enhances the EMT of CRC cells by modulating the expression of TIMP-2, MMP-2 and MMP-9.

\section{Acknowledgements}

This study was supported by Natural Science Foundation of Shandong Province (grant no. 2013ZRB14250).

\section{References}

1. World Health Organization (WHO): GLOBOCAN 2012: Estimated Cancer Incidence, Mortality and Prevalence Worldwide in 2012. http://globocan.iarc.fr/Pages/fact_sheets_ cancer.aspx. Accessed April 30, 2015.

2. Gupta GP and Massagué J: Cancer metastasis: Building a framework. Cell 127: 679-695, 2006.

3. Lamouille S, Subramanyam D, Blelloch R and Derynck R: Regulation of epithelial-mesenchymal and mesenchymal-epithelial transitions by microRNAs. Curr Opin Cell Biol 25: 200-207, 2013.

4. Schetter AJ, Okayama H and Harris CC: The role of microRNAs in colorectal cancer. Cancer J 18: 244-252, 2012.

5. Melton C, Judson RL and Blelloch R: Opposing microRNA families regulate self-renewal in mouse embryonic stem cells. Nature 463: 621-626, 2010
6. Yoo AS, Sun AX, Li L, et al: MicroRNA-mediated conversion of human fibroblasts to neurons. Nature 476: 228-231, 2011.

7. Rottiers V and Näär AM: MicroRNAs in metabolism and metabolic disorders. Nat Rev Mol Cell Biol 13: 239-250, 2012.

8. Rossi S, Di Narzo AF, Mestdagh P, et al: microRNAs in colon cancer: A roadmap for discovery. FEBS Lett 586: 3000-3007, 2012.

9. Bartels CL and Tsongalis GJ: MicroRNAs: Novel biomarkers for human cancer. Ann Biol Clin (Paris) 68: 263-272, 2010 (In French)

10. Tsuchida A, Ohno S, Wu W, et al: miR-92 is a key oncogenic component of the miR-17-92 cluster in colon cancer. Cancer Sci 102: 2264-2271, 2011.

11. Fan MQ, Huang CB, Gu Y, Xiao Y, Sheng JX and Zhong L: Decrease expression of microRNA-20a promotes cancer cell proliferation and predicts poor survival of hepatocellular carcinoma. J Exp Clin Cancer Res 32: 21, 2013.

12. Chang CC, Yang YJ, Li YJ, et al: MicroRNA-17/20a functions to inhibit cell migration and can be used a prognostic marker in oral squamous cell carcinoma. Oral Oncol 49: 923-931, 2013.

13. Chang Y, Liu C, Yang J, et al: MiR-20a triggers metastasis of gallbladder carcinoma. J Hepatol 59: 518-527, 2013.

14. Jeong WJ, Cha PH and Choi KY: Strategies to overcome resistance to epidermal growth factor receptor monoclonal antibody therapy in metastatic colorectal cancer. World J Gastroenterol 20: 9862-9871, 2014.

15. Schetter AJ, Leung SY, Sohn JJ, et al: MicroRNA expression profiles associated with prognosis and therapeutic outcome in colon adenocarcinoma. JAMA 299: 425-436, 2008.

16. Malzkorn B, Wolter M, Liesenberg F, et al: Identification and functional characterization of microRNAs involved in the malignant progression of gliomas. Brain Pathol 20: 539-550, 2010.

17. Volinia S, Calin GA, Liu CG, et al: A microRNA expression signature of human solid tumors defines cancer gene targets. Proc Natl Acad Sci USA 103: 2257-2261, 2006.

18. Yan H, Wu J, Liu W, Zuo Y, Chen S, Zhang S, Zeng M and Huang W: MicroRNA-20a overexpression inhibited proliferation and metastasis of pancreatic carcinoma cells. Hum Gene Ther 21: 1723-1734, 2010.

19. Diosdado B, van de Wiel MA, Terhaar Sive Droste JS, et al: MiR-17-92 cluster is associated with $13 \mathrm{q}$ gain and c-myc expression during colorectal adenoma to adenocarcinoma progression. Br J Cancer 101: 707-714, 2009.

20. Kalluri R and Weinberg RA: The basics of epithelial-mesenchymal transition. J Clin Invest 119: 1420-1428, 2009.

21. Zhu QC, Gao RY, Wu W and Qin HL: Epithelial-mesenchymal transition and its role in the pathogenesis of colorectal cancer. Asian Pac J Cancer Prev 14: 2689-2698, 2013.

22. Sun XJ, Zhang P, Li HH, Jiang ZW, Jiang CC and Liu H: Cisplatin combined with metformin inhibits migration and invasion of human nasopharyngeal carcinoma cells by regulating E-cadherin and MMP-9. Asian Pac J Cancer Prev 15: 4019-4023, 2014

23. Kessenbrock K, Plaks V and Werb Z: Matrix metalloproteinases: Regulators of the tumor microenvironment. Cell 141: 52-67, 2010.

24. Levi E, Fridman R, Miao HQ, Ma YS, Yayon A and Vlodavsky I: Matrix metalloproteinase 2 releases active soluble ectodomain of fibroblast growth factor receptor 1. Proc Natl Acad Sci USA 93: 7069-7074, 1996

25. Chen R, Cui J, Xu C, et al: The significance of MMP-9 over MMP-2 in HCC invasiveness and recurrence of hepatocellular carcinoma after curative resection. Ann Surg Oncol 19 (Suppl 3): S375-S384, 2012

26. Liu Y, Bi T, Shen G, et al: Lupeol induces apoptosis and inhibits invasion in gallbladder carcinoma GBC-SD cells by suppression of EGFR/MMP-9 signaling pathway. Cytotechnology: Jul 19, 2014 (Epub ahead of print)

27. Kallakury BV, Karikehalli S, Haholu A, Sheehan CE, Azumi N and Ross JS: Increased expression of matrix metalloproteinases 2 and 9 and tissue inhibitors of metalloproteinases 1 and 2 correlate with poor prognostic variables in renal cell carcinoma. Clin Cancer Res 7: 3113-3119, 2001.

28. Wang Z, Wang B, Shi Y, et al: Oncogenic miR-20a and miR-106a enhance the invasiveness of human glioma stem cells by directly targeting TIMP-2. Oncogene 34: 1407-1419, 2015. 